\title{
Accelerated Microwave Design Optimization With Tuning Space Mapping
}

\author{
Slawomir Koziel, Senior Member, IEEE, Jie Meng, John W. Bandler, Life Fellow, IEEE, \\ Mohamed H. Bakr, Member, IEEE, and Qingsha S. Cheng, Member, IEEE
}

\begin{abstract}
We introduce a tuning space-mapping technology for microwave design optimization. The general tuning space-mapping algorithm is formulated, which is based on a so-called tuning model, as well as on a calibration process that translates the adjustment of the tuning model parameters into relevant updates of the design variables. The tuning model is developed in a fast circuit-theory based simulator and typically includes the fine model data at the current design in the form of the properly formatted scattering parameter values. It also contains a set of tuning parameters, which are used to optimize the model so that it satisfies the design specification. The calibration process may involve analytical formulas that establish the dependence of the design variables on the tuning parameters. If the formulas are not known, the calibration process can be performed using an auxiliary space-mapping surrogate model. Although the tuning space mapping can be considered to be a specialized case of the standard space-mapping approach, it can offer even better performance because it enables engineers to exploit their experience within the context of efficient space mapping. Our approach is demonstrated using several microwave design optimization problems.
\end{abstract}

Index Terms-Computer-aided design (CAD), engineering optimization, space mapping, surrogate models, tuning.

\section{INTRODUCTION}

$\mathbf{S}$ PACE MAPPING is a widely recognized technique for optimization of expensive functions, also called "fine" models (typically implemented with CPU-intensive electromagnetic (EM) simulators), through iterative optimization and updating of the surrogate models, which are built using cheaper "coarse" models [1]-[6]. The coarse model, although less accurate, is still required to describe the fine model behavior relatively well, in which case, the space-mapping algorithm

Manuscript received June 02, 2008; revised October 13, 2008. First published January 23, 2009; current version published February 06, 2009 This work was supported in part by the Natural Sciences and Engineering Research Council of Canada (NSERC) under Grant RGPIN7239-06 and Grant STPGP336760-06 and by Bandler Corporation.

S. Koziel is with the School of Science and Engineering, Reykjavík University, IS-103 Reykjavík, Iceland (e-mail: koziel@ru.is).

J. Meng and Q. S. Cheng are with the Simulation Optimization Systems Research Laboratory, Department of Electrical and Computer Engineering, McMaster University, Hamilton, ON, Canada L8S 4K1 (e-mail: mengjie719@grads.ece.mcmaster.ca; chengq@mcmaster.ca).

J. W. Bandler is with the Simulation Optimization Systems Research Laboratory, Department of Electrical and Computer Engineering, McMaster University, Hamilton, ON, Canada L8S 4K1, and also with Bandler Corporation, Dundas, ON, Canada L9H 5E7 (e-mail: bandler@mcmaster.ca).

M. H. Bakr is with the Computational Electromagnetics Research Laboratory, Department of Electrical and Computer Engineering, McMaster University, Hamilton, ON, Canada L8S 4K1 (e-mail: mbakr@mail.ece.mcmaster.ca).

Color versions of one or more of the figures in this paper are available online at http://ieeexplore.ieee.org.

Digital Object Identifier 10.1109/TMTT.2008.2011313 typically provides satisfactory results after only a few evaluations of the fine model. Space mapping was originally applied in microwave engineering [7]-[11], but it has already proven its success in many other areas (e.g., [12]-[15]). Space mapping belongs to a broader family of surrogate-model-based optimization methods [16]-[21]; however, its distinctive feature is that the surrogate model is created through a physically based coarse model, but not by direct approximation of the available fine model data.

A number of papers cover different aspects of space mapping, including the development of new optimization algorithms [2]-[5], space-mapping-based modeling [22]-[24], neuro space mapping [25]-[28], theoretical foundations [4], [29], [30], etc.

The concept of tuning, also widely used in microwave engineering [31], [32], can be considered within the scope of the space-mapping approach. In our new tuning space-mapping algorithm, the surrogate model's role is taken by a so-called tuning model, which is constructed by introducing circuit-theory based components (e.g., capacitors, inductors, or coupled-line models) into the fine model structure, and parameters of these circuit components are chosen to be tunable. In each iteration, the tuning model is updated and optimized with respect to the tuning parameters. This process takes little CPU effort as the tuning model is typically implemented within a circuit simulator. With the optimal tuning parameters thus obtained, a calibration is needed to transform these tuning values into an appropriate modification of the design variables, which are then assigned to the fine model. The calibration process may involve analytical formulas (if a functional dependence of the design variables on the tuning parameters is known explicitly) or it may require an auxiliary model, typically a fast space-mapping surrogate. The tuning space-mapping iteration is repeated until the fine model response is sufficiently close to the design target. The structure of the tuning model, as well as a proper selection of the tuning elements, is crucial to the performance of the overall optimization process.

Approaches based on the idea of tuning space mapping have been proposed and applied in the microwave and RF arena, though they are not explicitly formulated in the space-mapping nomenclature. Rautio [31] introduced the tuning method developed by EPCOS for low-temperature co-fired ceramic (LTCC) design and stated that it is an effective technique that can be used for any RF design. Swanson [32] successfully applied the port tuning strategy to various microwave circuits such as combline filters.

In this paper, we give a formal description of our novel tuning space-mapping optimization algorithm that iteratively updates 


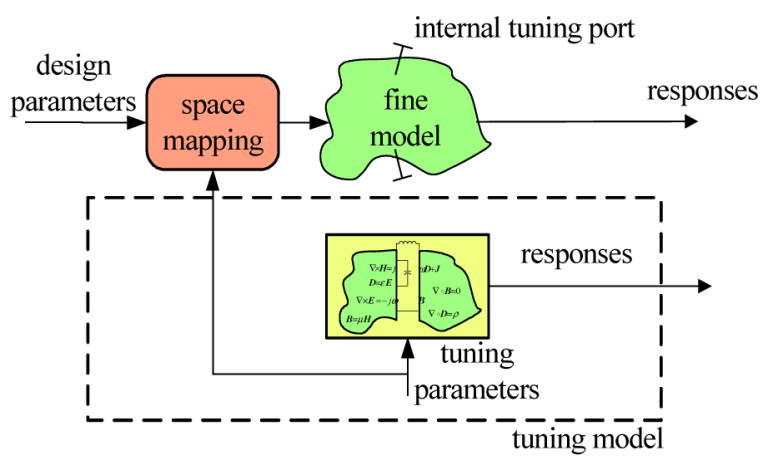

Fig. 1. Concept of the tuning model.

and optimizes a so-called tuning model in conjunction with a calibration procedure which translates the adjustments of the tuning parameters into relevant adjustments of the design variable values. The special case of this method, based on a spacemapping surrogate-model calibration, has been introduced in [33]. Here, we put the tuning space-mapping technique in a more general setting that also allows analytical calibration. We discuss the relation of the tuning space mapping to the standard space-mapping approach, provide its intuitive explanation through a simple microstrip line optimization problem, as well as discuss some practical issues of our method. Its robustness is demonstrated using several microwave design optimization problems.

It should be mentioned that Section II contains a general and detailed theory of the tuning space mapping technique. However, the concept and value of this approach can be captured by considering the examples of Section III. The interested reader can refer to Section II in order to find a rigorous description of the algorithm, as well as some important implementation details.

\section{TUNING SPACE MAPPING}

\section{A. Principle of the Tuning Space-Mapping Approach}

We are concerned with the following optimization problem:

$$
\boldsymbol{x}_{f}^{*}=\arg \min _{\boldsymbol{x}} U\left(\boldsymbol{R}_{f}(\boldsymbol{x})\right)
$$

where $\boldsymbol{R}_{f} \in R^{m}$ denotes the response vector of a fine model of the device of interest, $U$ is a merit function (e.g., a minimax function or a norm), $\boldsymbol{x}$ is a vector of design parameters, and $\boldsymbol{x}_{f}^{*}$ is the optimal solution to be determined; arg min in (1) emphasizes the fact that we are looking for the argument $\boldsymbol{x}$ that realizes the minimum of a function in question, here, $U\left(\boldsymbol{R}_{f}(\boldsymbol{x})\right)$.

The tuning space-mapping approach is an iterative optimization procedure that assumes the existence of a so-called tuning model $\boldsymbol{R}_{t}$, which is less accurate, but computationally much cheaper than the fine model. $\boldsymbol{R}_{t}$ contains relevant fine model data (typically a fine model response) at the current iteration point and tuning parameters (typically implemented through circuit elements inserted into tuning ports). The tunable parameters are adjusted so that the model $\boldsymbol{R}_{t}$ satisfies the design specifications. The conceptual illustration of the tuning model is shown in Fig. 1.

A certain relation (not necessarily analytical) between the parameters of the model $\boldsymbol{R}_{t}$ and the design variables is assumed so that the new design is obtained by translating the adjusted parameters of $\boldsymbol{R}_{t}$ into the corresponding design variable values using this very relation.

\section{B. Formulation of the Tuning Space-Mapping Algorithm}

The tuning space-mapping algorithm produces a sequence of points (design variable vectors) $\boldsymbol{x}^{(i)}, i=0,1, \ldots$ The iteration of the algorithm consists of two steps: optimization of the tuning model and a calibration procedure. First, the current tuning model $\boldsymbol{R}_{t}^{(i)}$ is built using fine model data at point $\boldsymbol{x}^{(i)}$. In general, because the fine model has undergone a disturbance, the tuning model response may not agree with the response of the fine model at $\boldsymbol{x}^{(i)}$ even if the values of the tuning parameters $\boldsymbol{x}_{t}$ are zero so that these values must be adjusted to, say, $\boldsymbol{x}_{t .0}^{(i)}$ in order to obtain alignment as follows[33]:

$$
\boldsymbol{x}_{t .0}^{(i)}=\arg \min _{\boldsymbol{x}_{t}}\left\|\boldsymbol{R}_{f}\left(\boldsymbol{x}^{(i)}\right)-\boldsymbol{R}_{t}^{(i)}\left(\boldsymbol{x}_{t}\right)\right\| .
$$

In the next step, we optimize $\boldsymbol{R}_{t}^{(i)}$ to have it meet the design specifications. We obtain the optimal values of the tuning parameters $\boldsymbol{x}_{t .1}^{(i)}$ as follows [33]:

$$
\boldsymbol{x}_{t .1}^{(i)}=\arg \min _{\boldsymbol{x}_{t}} U\left(\boldsymbol{R}_{t}^{(i)}\left(\boldsymbol{x}_{t}\right)\right) .
$$

Having $\boldsymbol{x}_{t .1}^{(i)}$, we perform the calibration procedure to determine changes in the design variables that yield the same change in the calibration model response as that caused by $x_{t .1}^{(i)}-x_{t .0}^{(i)}$.

The calibration, denoted here as $C$, can be formally defined as

$$
\boldsymbol{x}^{(i+1)}=C\left(\boldsymbol{x}^{(i)}, \boldsymbol{x}_{t .1}^{(i)}, \boldsymbol{x}_{t .0}^{(i)}\right)
$$

where $x^{(i+1)}$ is the new design determined by the current design $\boldsymbol{x}^{(i)}$, the optimal tuning parameters $\boldsymbol{x}_{t .1}^{(i)}$ from (3), and the alignment tuning parameters $\boldsymbol{x}_{t .0}^{(i)}$ from (2).

The calibration process $C$ can be generally written as

$$
\boldsymbol{x}^{*}=C\left(\boldsymbol{x}, \boldsymbol{x}_{t}^{*}, \boldsymbol{x}_{t}\right)
$$

and describes the relation between the design and tuning parameters. In particular, $\boldsymbol{x}^{*}$ denotes the vector of the design parameters that corresponds to a change of the tuning parameters from $\boldsymbol{x}_{t}$ to $\boldsymbol{x}_{t}^{*}$ with $\boldsymbol{x}$ being the initial values of the design parameters.

\section{Calibration Process}

Calibration can be realized in three possible ways.

Direct Calibration: This is the simplest calibration method where the relation between the design variables and the tuning parameters is assumed to be an identity function, i.e., we have $\boldsymbol{x}^{(i+1)}=\boldsymbol{x}^{(i)}+\left(\boldsymbol{x}_{t .1}^{(i)}-\boldsymbol{x}_{t .0}^{(i)}\right)$. This calibration method can be 
useful if the tuning components are closely related to the elements of the microwave structure represented by the design variables (e.g., microstrip line models as tuning elements and actual microstrip line lengths as design variables).

Analytical Calibration: In some cases there is a formula $C$ that establishes an analytical relation between the design variables and the tuning parameters so that (5) is realized simply by applying this formula. For example, the calibration formula may be just a linear function so that $\boldsymbol{x}^{(i+1)}=\boldsymbol{x}^{(i)}+\boldsymbol{s}^{(i)} *\left(\boldsymbol{x}_{t .1}^{(i)}-\boldsymbol{x}_{t .0}^{(i)}\right)$, where $\boldsymbol{s}^{(i)}$ is a real vector and $*$ denotes a component-wise multiplication.

Equivalent Circuit Calibration: In many cases, however, such a formula is not known and calibration is performed using an auxiliary model $\boldsymbol{R}_{c}$, here called a calibration model [33]. Similar to $\boldsymbol{R}_{t}, \boldsymbol{R}_{c}$ is also assumed to be computationally cheap. Normally, $\boldsymbol{R}_{c}$ is dependent on three sets of variables: design parameters, tuning parameters (which are actually the same parameters as the ones used in $\boldsymbol{R}_{t}$ ), and space-mapping parameters that are adjusted using the usual parameter extraction process [4] in order to have model $\boldsymbol{R}_{c}$ meet certain matching conditions. Typically, the model $\boldsymbol{R}_{c}$ is a standard space-mapping surrogate (i.e., a coarse model composed with suitable transformations) enhanced by the same or corresponding tuning elements as the model $\boldsymbol{R}_{t}$.

The calibration process with the model $\boldsymbol{R}_{c}$ is as follows. We first adjust the space-mapping parameters of the calibration model to obtain a match with the fine model response at $\boldsymbol{x}^{(i)}$

$$
\boldsymbol{p}^{(i)}=\arg \min _{\boldsymbol{p}}\left\|\boldsymbol{R}_{f}\left(\boldsymbol{x}^{(i)}\right)-\boldsymbol{R}_{c}\left(\boldsymbol{x}^{(i)}, \boldsymbol{p}, \boldsymbol{x}_{t .0}^{(i)}\right)\right\| .
$$

The calibration model is then optimized with respect to the design variables in order to obtain the next iteration point $\boldsymbol{x}^{(i+1)}$

$$
\boldsymbol{x}^{(i+1)}=\arg \min _{\boldsymbol{x}}\left\|\boldsymbol{R}_{t}^{(i)}\left(\boldsymbol{x}_{t .1}^{(i)}\right)-\boldsymbol{R}_{c}\left(\boldsymbol{x}, \boldsymbol{p}^{(i)}, \boldsymbol{x}_{t .0}^{(i)}\right)\right\|
$$

Note that we use $\boldsymbol{x}_{t .0}^{(i)}$ in (6), which corresponds to the state of the tuning model after performing the alignment procedure (2), and $x_{t .1}^{(i)}$ in (7), which corresponds to the optimized tuning model [cf. (3)]. Thus, (6) and (7) allow us to find the change of design variable values $x^{(i+1)}-x^{(i)}$ necessary to compensate the effect of changing the tuning parameters from $x_{t .0}^{(i)}$ to $x_{t .1}^{(i)}$.

There are a number of other possible approaches. A notable example is a mixed procedure, where an analytical calibration is performed with respect to some of the tuning parameters, while the rest of the parameters are calibrated using a calibration model as in (6) and (7).

\section{Relation to Standard Space Mapping}

The standard space mapping [4] is formulated as

$$
\boldsymbol{x}^{(i+1)}=\arg \min _{\boldsymbol{x}} U\left(\boldsymbol{R}_{\boldsymbol{s}}\left(\boldsymbol{x}, \boldsymbol{p}^{(i)}\right)\right)
$$

where $\boldsymbol{R}_{s}\left(\boldsymbol{x}, \boldsymbol{p}^{(i)}\right)$ is a surrogate model at iteration $i$ with spacemapping parameters $\boldsymbol{p}^{(i)}$ obtained from the following parameter extraction process:

$$
\boldsymbol{p}^{(i)}=\arg \min _{\boldsymbol{p}} \sum_{k=0}^{i} w_{i . k}\left\|\boldsymbol{R}_{f}\left(\boldsymbol{x}^{(k)}\right)-\boldsymbol{R}_{s}\left(\boldsymbol{x}^{(k)}, \boldsymbol{p}\right)\right\|
$$

with $w_{i . k}$ being weighting factors [4].

To reveal the relationship between tuning space mapping and standard space mapping, we assume that the general calibration function (5) is invertible with respect to its second argument $\left(\boldsymbol{x}_{t}^{*}\right)$ for any fixed first and third arguments ( $\boldsymbol{x}$ and $\boldsymbol{x}_{t}$, respectively). At the $i$ th iteration, given that $\boldsymbol{x}$ and $\boldsymbol{x}_{t}$ are, respectively, fixed at $\boldsymbol{x}^{(i)}$ and $\boldsymbol{x}_{t .0}^{(i)}$, we have the "inverted" calibration function $C^{-1}$ as

$$
x_{t}=C^{-1}\left(x^{(i)}, x, x_{t .0}^{(i)}\right) .
$$

Using (10), we can substitute $\boldsymbol{x}_{t}$ in $\boldsymbol{R}_{t}\left(\boldsymbol{x}_{t}\right)$ with a function of $\boldsymbol{x}$ and $\boldsymbol{x}_{t .0}^{(i)}$. Thus, we can define a surrogate model $\boldsymbol{R}_{s}$ as

$$
\boldsymbol{R}_{s}\left(\boldsymbol{x}, \boldsymbol{x}_{t .0}^{(i)}\right)=\boldsymbol{R}_{t}^{(i)}\left(C^{-1}\left(\boldsymbol{x}^{(i)}, \boldsymbol{x}, \boldsymbol{x}_{t .0}^{(i)}\right)\right)
$$

where $\boldsymbol{x}_{t .0}^{(i)}$ acts as the surrogate model parameters (equivalent to $p^{(i)}$ in (8)), while $\boldsymbol{x}^{(i)}$ is a constant at the $i$ th iteration. By substituting (10) into (2), we can determine $\boldsymbol{x}_{t .0}^{(i)}$ as follows:

$$
\boldsymbol{x}_{t .0}^{(i)}=\arg \min _{\boldsymbol{x}_{t .0}}\left\|\boldsymbol{R}_{f}\left(\boldsymbol{x}^{(i)}\right)-\boldsymbol{R}_{t}^{(i)}\left(C^{-1}\left(\boldsymbol{x}^{(i)}, \boldsymbol{x}^{(i)}, \boldsymbol{x}_{t .0}\right)\right)\right\| .
$$

Through comparing (8) with (11) and (9) with (12), we can clearly recognize tuning space mapping as a specialized case of standard space mapping. To be more specific, (12) is a special case of (9) with $w_{i . k}=1$ for $k=i$, and $w_{i . k}=0$ for $k=0,1, \ldots, i-1$, i.e., tuning space mapping uses only the last iteration point in the parameter extraction procedure. Furthermore, (11) shows that the tuning model is simply a special type of surrogate model. At the same time, tuning space mapping allows greater flexibility in terms of the surrogate model, which may, in general, involve any relation between the tuning parameters and design variables.

It should be noted that the tuning space-mapping method exploits both the standard space-mapping optimization, classical circuit theory, and EM theory, as well as practical experience. For example, in a physics-based simulation according to classical EM theory, design parameters such as physical length and width of a microstrip line can be mapped to a "tuning component" such as an inductor or capacitor. The calibration process then transfers the tuning parameters to physical design parameters, which can be achieved by taking advantage of classical theory and engineering experience.

\section{E. Illustration of the Tuning Space-Mapping Algorithm}

In order to illustrate and clarify our tuning space mapping algorithm, we use a simple example of a microstrip transmission line [31]. The fine model is implemented in Sonnet $\boldsymbol{e m}$ [34] (Fig. 2), and the fine model response is taken as the inductance of the line as a function of the line's length. The original length 


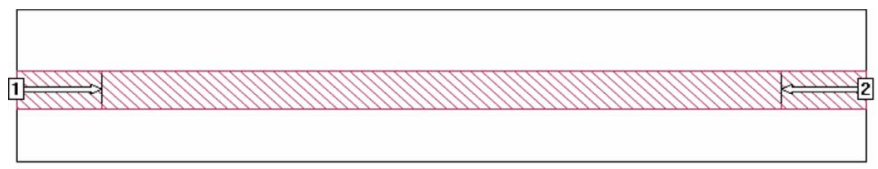

Fig. 2. Original structure of microstrip line in Sonnet.

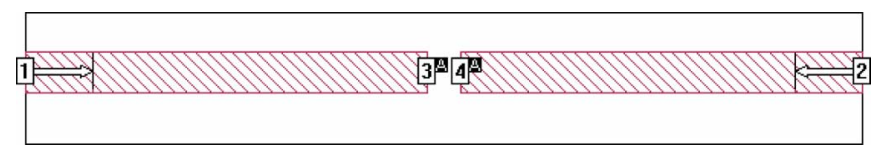

Fig. 3. Microstrip line under test after being divided and with the inserted co-calibrated ports.

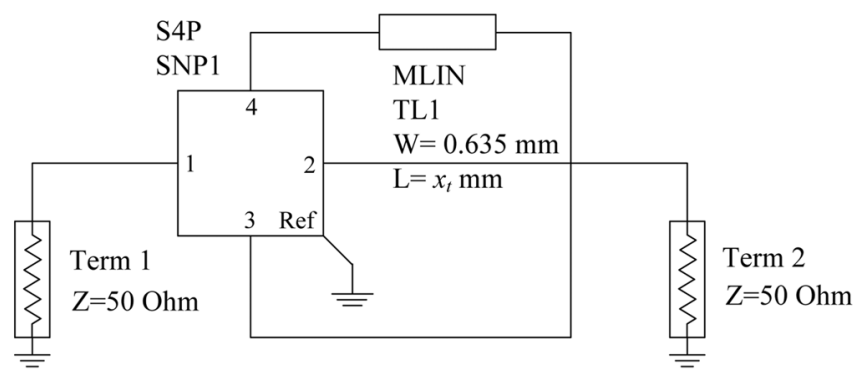

(a)

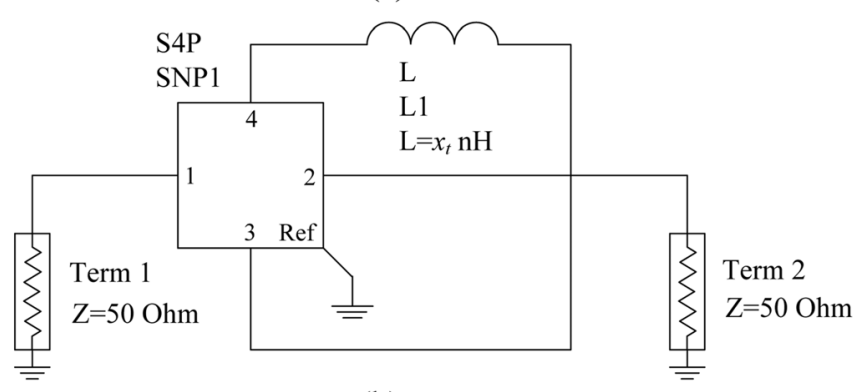

(b)

Fig. 4. Microstrip line design problem. (a) Tuning model for direct calibration method. (b). Tuning model for analytical and equivalent circuit calibration.

of the line is chosen to be $\boldsymbol{x}^{(0)}=10.16 \mathrm{~mm}$ (400 mil) with a width of $0.635 \mathrm{~mm}$. We use a cell size of $0.01 \times 0.635 \mathrm{~mm}$. A substrate with thickness $H=0.635 \mathrm{~mm}$ and $\varepsilon_{r}=9.8$ is used. Our goal is to find a length of line such that the corresponding inductance is $6.5 \mathrm{nH}$ at $300 \mathrm{MHz}$. The Sonnet $\boldsymbol{e m}$ simulation at $\boldsymbol{x}^{(0)}$ gives the value of $4.52 \mathrm{nH}$, i.e., $\boldsymbol{R}_{f}\left(\boldsymbol{x}^{(0)}\right)=4.52 \mathrm{nH}$.

We use the tuning space mapping algorithm of Section II-B. The tuning model $\boldsymbol{R}_{t}$ is developed by dividing the structure in Fig. 2 into two separate parts and adding the two tuning ports, as shown in Fig. 3. A tuning element (e.g., a small inductor or a microstrip line segment) is then inserted between these ports. Note that the new version of Sonnet $\boldsymbol{e m}$ allows so-called co-calibrated ports [34]. The ports allow an "infinitesimal" gap (normally one cell size wide) to be inserted into a microwave structure. A pair of such ports can then be mounted on the edges of the gap. The multiport structure is then simulated electromagnetically in Sonnet $\boldsymbol{e m}$ and then connected in the circuit simulator. The partition and gap are compensated by Sonnet $\boldsymbol{e m}$ so that the impact on the simulation results is negligible.

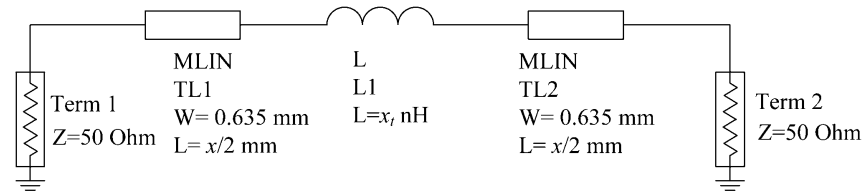

Fig. 5. Calibration model for the microstrip line design problem.

Tuning Space Mapping Using Direct Calibration: The tuning model is implemented in Agilent ADS [35] and shown in Fig. 4(a). The model contains the fine model data at the initial design in the form of the S4P element (4 being the number of ports), as well as the tuning element (microstrip line segment). Due to Sonnet's co-calibrated ports, a "perfect" agreement between the fine and tuning model still leaves $\boldsymbol{x}_{t .0}^{(0)}$ at a small value of $0.01 \mathrm{~mm}$.

Next, we optimize the tuning model to meet our target inductance $6.5 \mathrm{nH}$. The optimized value of the tuning microstrip length is $\boldsymbol{x}_{t .1}^{(0)}=4.52 \mathrm{~mm}$.

Now, we need to perform the calibration step. Here, because the tuning element is a microstrip line segment, we use a direct calibration, i.e., we simply assume that $\boldsymbol{x}^{(1)}=\boldsymbol{x}^{(0)}+\left(\boldsymbol{x}_{t .1}^{(0)}-\right.$ $\left.\boldsymbol{x}_{t .0}^{(0)}\right)$, which gives $\boldsymbol{x}^{(1)}=14.67 \mathrm{~mm}$. The fine model response at $\boldsymbol{x}^{(1)}$ obtained by Sonnet $\boldsymbol{e m}$ simulation is $6.59 \mathrm{nH}$. The second iteration of tuning space mapping gives $\boldsymbol{x}^{(2)}=14.48 \mathrm{~mm}$; the corresponding line inductance reaches our goal of $6.50 \mathrm{nH}$.

Tuning Space Mapping Using Analytical Calibration: In this case, the tuning model is implemented in Agilent ADS [35] and is shown in Fig. 4(b). The model contains the fine model data at the initial design in the form of the S4P element (4 being the number of ports), as well as the tuning element (inductor). Due to Sonnet's co-calibrated ports, a "perfect" match between the fine and tuning model responses is obtained with a small value of $\boldsymbol{x}_{t .0}^{(0)}=0.004$.

Next, we optimize the tuning model to meet our target inductance $6.5 \mathrm{nH}$. The optimized value of the tuning inductance is $\boldsymbol{x}_{t .1}^{(0)}=1.94 \mathrm{nH}$.

Now, we need to perform the calibration step. In our example, it is easy to develop an analytical calibration formula because we can propose a linear dependence between the microstrip length $\boldsymbol{x}$ and the inductance of the tuning element $\boldsymbol{x}_{t}$. The proportionality coefficient is equal $\boldsymbol{s}^{(0)}=\boldsymbol{x}^{(0)} / \boldsymbol{R}_{f}\left(\boldsymbol{x}^{(0)}\right)=$ $10.16 \mathrm{~mm} / 4.517 \mathrm{nH}=2.249 \mathrm{~mm} \cdot \mathrm{nH}^{-1}$. Thus, we have $\boldsymbol{x}^{(1)}=\boldsymbol{x}^{(0)}+\boldsymbol{s}^{(0)} \cdot\left(\boldsymbol{x}_{t .1}^{(0)}-\boldsymbol{x}_{t .0}^{(0)}\right)$, which gives $\boldsymbol{x}^{(1)}=14.51 \mathrm{~mm}$. The fine model response at $\boldsymbol{x}^{(1)}$ obtained by Sonnet $\boldsymbol{e m}$ simulation is $6.51 \mathrm{nH}$, which is already acceptable. After another two iterations, the tuning space mapping gives $\boldsymbol{x}^{(3)}=14.48 \mathrm{~mm}$; the corresponding line inductance is again $6.50 \mathrm{nH}$.

Tuning Space Mapping Using Equivalent Circuit Calibration: Again, we use the tuning model shown in Fig. 4(b). The calibration is now based on an equivalent circuit space-mapping surrogate model. Agreement between the fine and tuning model responses gives an $\boldsymbol{x}_{t .0}^{(0)}$ of $0.004 \mathrm{nH}$. We consider the calibration model shown in Fig. 5 in which the relative permeability (initially 1 ) of the microstrip element is used as a space mapping parameter $\boldsymbol{p}$. The value of this parameter is adjusted using (6) to 1.04 so that the response of the calibration model 
is $4.52 \mathrm{nH}$ at $10.16 \mathrm{~mm}$, i.e., it agrees with the fine model response at $\boldsymbol{x}^{(0)}$. Now, we need to obtain the new value of the microstrip length, which is done according to (7). In particular, we optimize $\boldsymbol{x}$ (length of the line) with the tuning inductance set to $\boldsymbol{x}_{t .0}^{(0)}=0.004 \mathrm{nH}$ to match the total inductance of the calibration model to the optimized tuning model response, $6.5 \mathrm{nH}$. The result is $\boldsymbol{x}^{(1)}=14.48 \mathrm{~mm}$; the fine model response at $\boldsymbol{x}^{(1)}$ obtained by Sonnet $\boldsymbol{e m}$ simulation is $6.50 \mathrm{nH}$. There is no need for a second iteration.

\section{F. Practical Aspects of Tuning Space Mapping}

As mentioned in Section I, the structure of the tuning model, as well as a proper selection of tuning elements are crucial for the performance of the overall optimization process. With a properly chosen tuning model, it is possible to obtain excellent results even faster than with the standard space-mapping approach. It is not uncommon that the design specifications are satisfied after a single iteration of the tuning space-mapping algorithm. Tuning space mapping appears more physically based than regular space mapping, and thus, should be readily accepted by engineers.

Although it is difficult to provide detailed guidelines on designing the tuning and/or calibration model, as both may be heavily dependent on a particular problem, there are several recommendations that may be useful.

Probably the best way to construct the tuning model at any given iteration point (design) is to "cut" it in the sense explained in the microstrip line example of Section II-E, obtain the $S$-parameters of the resulting multiport structure, and then endow the element representing these parameters (e.g., the S4P element in an ADS schematic) with all the necessary tuning elements. This procedure has been illustrated in Section II-E; more involved examples are provided in Section III.

The calibration model should actually mimic the structure of the tuning model so that the multiport structure described in the previous paragraph is replaced by any reasonable circuit equivalent, while the topology of the tuning part remains unchanged. In this way, there is a one-to-one correspondence between the tuning parameters of the tuning model and the ones of the calibration model. Other realizations are also conceivable, but the one described above seems the most reasonable. Again, the concept has been explained in Section II-E. The remaining part of the paper contains more examples.

As mentioned earlier, the choice of tuning parameters requires appropriate knowledge in the field so no general guidelines can be given in this respect. The important prerequisite is, however, that both the tuning model optimization problem (3) and the calibration process (5) have unique solutions. In the case of surrogate-based calibration, both the parameter extraction problem (6) and (7) should also have unique solutions. In general, this requires that the number of tuning parameters is the same as the number of design variables. We also recommend that the number of space-mapping parameters of the calibration model is preferably the same and not larger than the number of design variables.

It should be mentioned that at the current stage it is not clear how to apply the tuning space mapping to tune cross-sectional dimensions such as microstrip widths or substrate heights. Thus

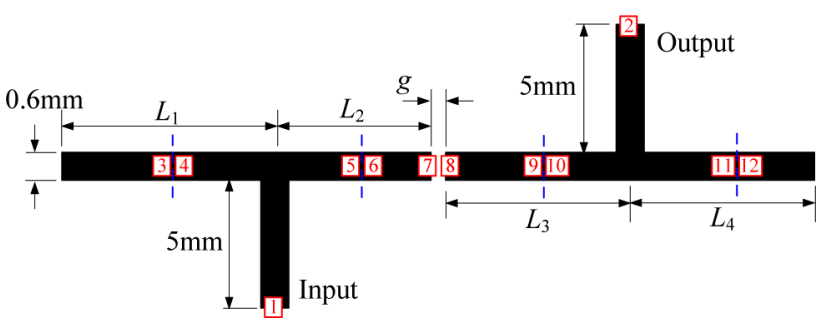

Fig. 6. Microstrip bandpass filter: physical structure [36].

far, the best way to optimize such parameters is still the standard space mapping approach.

\section{EXAMPLES}

\section{A. Microstrip Bandpass Filter [36]}

In the first example, we consider the design of a microstrip bandpass filter [36]. In order to illustrate how tuning elements are chosen and translated to physical dimensions, we implement this design problem in two different ways. Two sets of tuning parameters are chosen to construct the tuning models, and two alternative analytical calibration methods are demonstrated.

Problem Description: As shown in Fig. 6, $L_{1}, L_{2}, L_{3}$, and $L_{4}$ are the lengths of the microstrip-line sections and $g$ is the gap in the middle between the two central adjacent microstrip lines. The width $W$ is the same for all the lines, as well as for the input and output microstrip lines, whose length is $L_{0}$. A substrate with thickness $H$ and dielectric constant $\varepsilon_{r}$ is used. Both the dielectric losses and the metallization losses are considered to be zero.

The design parameters are $\boldsymbol{x}=\left[\begin{array}{lllll}L_{1} & L_{2} & L_{3} & L_{4} & g\end{array}\right]^{T}$. Other parameters are fixed at $L_{0}=3 \mathrm{~mm}, W=1 \mathrm{~mm}, H=0.66 \mathrm{~mm}$, and $\varepsilon_{r}=9.0$. The design specifications are

$$
\begin{aligned}
& \left|S_{21}\right| \leq-20 \mathrm{~dB}, \quad \text { for } 4.5 \mathrm{GHz} \leq \omega \leq 4.7 \mathrm{GHz} \\
& \left|S_{21}\right| \geq-3 \mathrm{~dB}, \quad \text { for } 4.9 \mathrm{GHz} \leq \omega \leq 5.1 \mathrm{GHz} \\
& \left|S_{21}\right| \leq-20 \mathrm{~dB}, \quad \text { for } 5.3 \mathrm{GHz} \leq \omega \leq 5.5 \mathrm{GHz} .
\end{aligned}
$$

Sonnet's $\boldsymbol{e m}$ is used as an EM simulator to evaluate the fine model. Agilent Technologies' ADS provides both tuning model and optimization tools; its component " $N$-port $S$-parameter file" enables simulated $S$-parameters to be imported in Touchstone file format from Sonnet's $\boldsymbol{e m}$.

The essential step is to construct the tuning model. Firstly, in Sonnet, we divide each microstrip polygon in the middle and insert co-calibrated ports at each pair of adjacent edges. The entire structure is then simulated in Sonnet's $\mathbf{e m}$ and the subsequent S12P data file is loaded into a 12-port $S$-parameter file component in ADS. After that, appropriate circuit components are chosen and attached onto the corresponding tuning ports of the 12-port $S$-parameter component.

Tuning Space Mapping With Analytical Calibration Method: We make the most intuitive choice of the tuning elements: circuit microstrip-line components and a gap component, as shown in Fig. 7. The lengths of the microstrip-line components and the spacing of the gap component are our tuning variables, i.e., $\boldsymbol{x}_{t}=\left[\begin{array}{lllll}L_{t 1} & L_{t 2} & L_{t 3} & L_{t 4} & g_{t}\end{array}\right]^{T} \mathrm{~mm}$. 


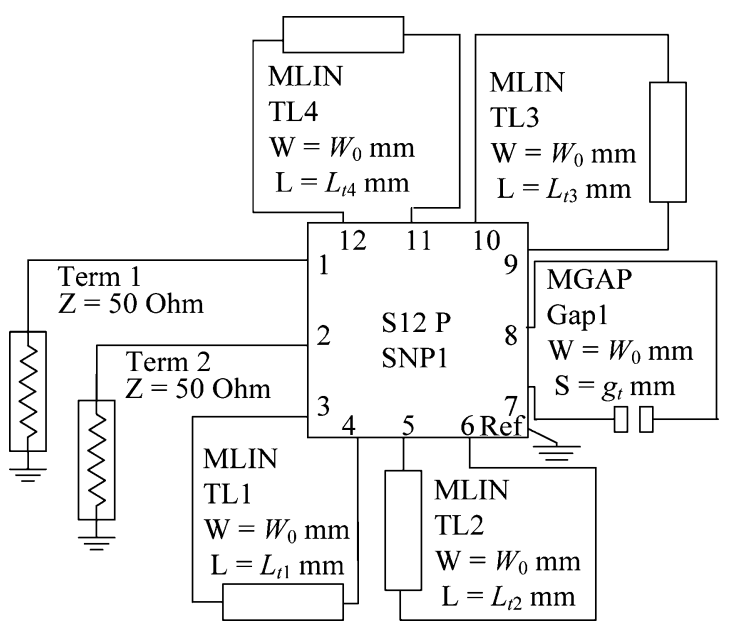

Fig. 7. Microstrip bandpass filter: tuning model (Agilent Technologies’ ADS).

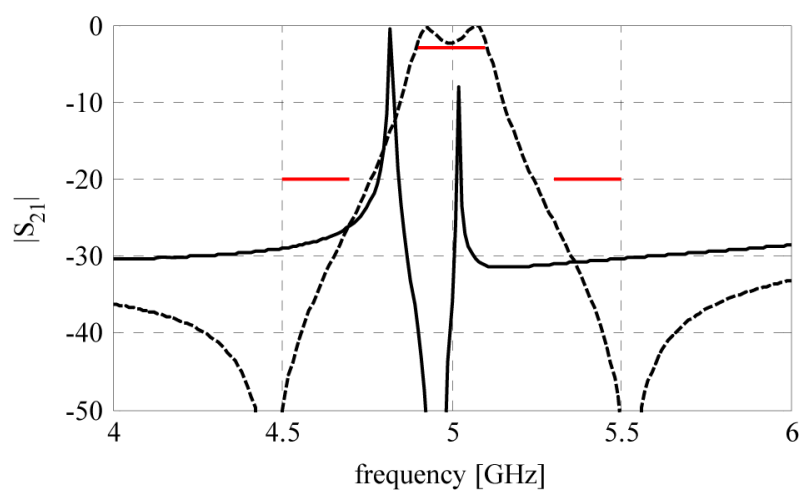

Fig. 8. Microstrip bandpass filter: fine model response at the initial design (solid line) and the response of the optimized tuning model (dashed line).

We choose $\boldsymbol{x}^{(0)}=\left[\begin{array}{lllll}6.00 & 6.00 & 6.00 & 6.00 & 0.08\end{array}\right]^{T} \mathrm{~mm}$ as an initial guess of the design parameters. The misalignment between the fine model (Sonnet $\boldsymbol{e m}$ ) response and the tuning model response with the tuning parameters set to zero is sufficiently small so that it can be ignored. Thus, an alignment process is not necessary and it is obvious that $\boldsymbol{x}_{t .0}^{(i)}=\left[\begin{array}{lllll}0 & 0 & 0 & 0 & 0\end{array}\right]^{T} \mathrm{~mm}$.

The optimization process with respect to the tuning parameters is implemented in ADS, which is aimed to satisfy the design specifications. The tuning parameters obtained with (3) are

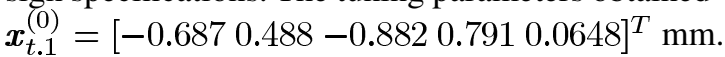

Note that some of the parameters take negative values, which is permitted in ADS. The tuning optimization result and the response of the fine model with the initial guess are shown in Fig. 8.

The calibration process in this example is straightforward: the optimal values of the tuning parameters are converted to the adjustments of the design parameters assuming an identity relation, i.e., the optimized lengths of the small microstrip components are exactly the changes of the lengths of the microstrip sections in the fine model, and the optimal spacing of the gap component is directly taken as the adjustment of the gap in the EM structure. More formally, we assume a calibration formula of the form $\boldsymbol{x}^{(i+1)}=\boldsymbol{x}^{(i)}+\boldsymbol{s} *\left(\boldsymbol{x}_{t .1}^{(i)}-\boldsymbol{x}_{t .0}^{(i)}\right)$, where $*$ denotes a component-wise multiplication. Coefficient vector $\boldsymbol{s}$ is defined as $\boldsymbol{s}=\left[\begin{array}{lllll}s_{1} & s_{2} & s_{3} & s_{4} & s_{5}\end{array}\right]^{T}$ with all $s_{k}, k=1, \ldots, 5$, equal to 1 .

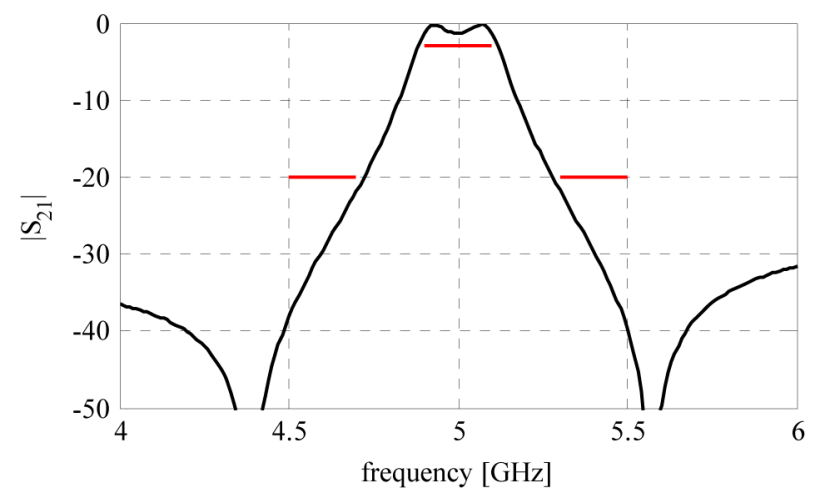

Fig. 9. Microstrip bandpass filter: fine model response $\left(\left|S_{21}\right|\right.$ obtained with Sonnet $\boldsymbol{e m}$ ) at the final design.

TABLE I

SONNET $\boldsymbol{E} \boldsymbol{M}$ Design Parameter VALues OF THE MICROSTRIP BANDPASS FILTER

\begin{tabular}{cccc}
\hline $\begin{array}{c}\text { Design } \\
\text { Parameters }\end{array}$ & Initial Solution & $\begin{array}{c}\text { Solution after } \\
\text { the First Iteration }\end{array}$ & $\begin{array}{c}\text { Solution after } \\
\text { the Second Iteration }\end{array}$ \\
\hline$L_{1}$ & $6.00 \mathrm{~mm}$ & $5.313 \mathrm{~mm}$ & $5.449 \mathrm{~mm}$ \\
$L_{2}$ & $6.00 \mathrm{~mm}$ & $6.488 \mathrm{~mm}$ & $6.363 \mathrm{~mm}$ \\
$L_{3}$ & $6.00 \mathrm{~mm}$ & $5.118 \mathrm{~mm}$ & $5.316 \mathrm{~mm}$ \\
$L_{4}$ & $6.00 \mathrm{~mm}$ & $6.791 \mathrm{~mm}$ & $6.667 \mathrm{~mm}$ \\
$g$ & $0.08 \mathrm{~mm}$ & $0.145 \mathrm{~mm}$ & $0.153 \mathrm{~mm}$ \\
\hline \hline
\end{tabular}

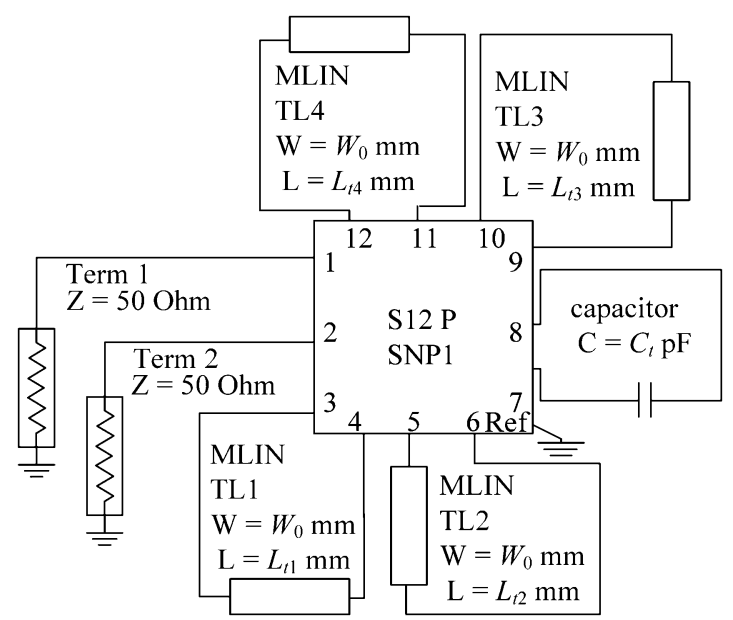

Fig. 10. Microstrip bandpass filter: alternative tuning model (Agilent Technologies' ADS).

After the first iteration, the new design $\boldsymbol{x}^{(1)}=$ $\left[\begin{array}{lllll}5.313 & 6.488 & 5.118 & 6.791 & 0.145\end{array}\right]^{T} \mathrm{~mm}$ has already satisfied the design specifications. A better solution $\boldsymbol{x}^{(2)}=\left[\begin{array}{l}5.4496 .3635 .3166 .6670 .153\end{array}\right]^{T} \mathrm{~mm}$ is obtained after the second tuning space-mapping iteration. Fig. 9 shows the fine model response at $\boldsymbol{x}^{(2)}$. The values of the design variables are summarized in Table I.

Tuning Space Mapping With Analytical Calibration and Alternative Tuning Model: The basic algorithm and concepts of tuning space-mapping method are demonstrated in the above design process. In some cases, however, it is impractical to find circuit components, whose characteristic parameters can be directly converted to the dimensions of fine model structures.

To illustrate the case where a nontrivial calibration formula is required, we again consider our bandpass filter example; 
however, we use the slightly different tuning model shown in Fig. 10. The only difference with the model in Fig. 7 is the tuning component for tuning the gap. In ADS, circuit-based microstrip components are still used to tune the lengths of the microstrip sections, while a capacitor is used instead of the gap component to calibrate the gap in the EM structure. These tuning components are then inserted between the tuning ports. Correspondingly, the lengths of the microstrip sections and the capacitance of the capacitor are our tuning variables, i.e., $x_{t}=\left[\begin{array}{lllll}L_{t 1} & L_{t 2} & L_{t 3} & L_{t 4} & C_{t}\end{array}\right]^{T}$ (lengths in millimeters, capacitance in picofarads).

The initial design is again set as $\boldsymbol{x}^{(0)}=$ $\left[\begin{array}{lllll}6.00 & 6.00 & 6.00 & 6.00 & 0.08\end{array}\right]^{T} \mathrm{~mm}$. In this case, even though the tuning elements have been inserted using the co-calibrated ports, there is still some misalignment between the fine model (Sonnet $\boldsymbol{e m}$ ) response and the tuning model response with the tuning elements set to be zero. Therefore, the alignment process (2) produces nontrivial values of $\boldsymbol{x}_{t .0}^{(0)}=\left[\begin{array}{lllll}-0.022 & 0.082 & 0.101 & 0.004 & 0.041\end{array}\right]^{T}$. The tuning parameters obtained with (3) are $\boldsymbol{x}_{t .1}^{(0)}=\left[\begin{array}{lll}-0.690 & 0.500-0.8270 .7920 .007\end{array}\right]^{T}$.

To translate the tuning parameters to design parameters, we again take a linear calibration formula of the form $x^{(i+1)}=$ $\boldsymbol{x}^{(i)}+s *\left(\boldsymbol{x}_{t .1}^{(i)}-\boldsymbol{x}_{t .0}^{(i)}\right)$, where $*$ denotes a component-wise multiplication with $\boldsymbol{s}=\left[s_{1} s_{2} s_{3} s_{4} s_{5}\right]^{T}$. Here, $s_{k}, k=1,2,3,4,5$ relate the changes of the design variables $L_{k}, k=1,2,3,4$, and $g$ to the changes of the corresponding tuning parameters, $L_{t k}, k=1,2,3,4$, and $C_{t}$.

This time, we do not assume $s_{k}=1$, but we actually find the relation between the change of the tuning component and the change of the corresponding design variable by means of simple auxiliary experiments.

The values of $s_{1}$ to $s_{4}$ were obtained from the setting shown in Figs. 2-5 with the inductor $L$ replaced by the ideal microstrip component in the ADS model. The simulations of the perturbed EM microstrip structure are implemented at only a couple of frequency points in Sonnet $\boldsymbol{e m}$. The corresponding perturbation of the ADS microstrip model that causes the same change of the line response is found using the alignment process (2). In the alignment process, the phase of $S_{21}$ is chosen as the response, as this is the parameter that is clearly length dependent. The obtained value of calibration coefficients is 1.0106 . It is close to 1 as expected.

The coefficient $s_{5}$, which relates the design variable $g$ with the tuning capacitance $C_{t}$, is obtained in the following way. The initial design $\boldsymbol{x}^{(0)}$ is perturbed with respect to $g$ by $\Delta g=$ $0.02 \mathrm{~mm}$, and the corresponding change $\Delta C_{t}$ of $C_{t}$ is found for which the fine model response at the above perturbed design is matched by the tuning model response at $\boldsymbol{x}_{t .0}^{(0)}$ perturbed by $\Delta C_{t}$. In our case, $\Delta C_{t}=0.0034 \mathrm{pF}$ so that $s_{5}=\Delta g / \Delta C_{t}=$ $-5.88 \mathrm{~mm} / \mathrm{pF}$.

The new design obtained with our calibration formula is $\boldsymbol{x}^{(1)}=\left[\begin{array}{lllll}5.333 & 6.429 & 5.118 & 6.971 & 0.041\end{array}\right] \mathrm{mm}$. Although it satisfies the design specifications, we perform a second tuning space-mapping iteration, which gives $x^{(2)}=\left[\begin{array}{lllll}5.362 & 6.462 & 5.210 & 6.785 & 0.120\end{array}\right]^{T}$ mm. Fig. 11 shows the fine model response at $\boldsymbol{x}^{(2)}$. The values of the design variables are summarized in Table II.

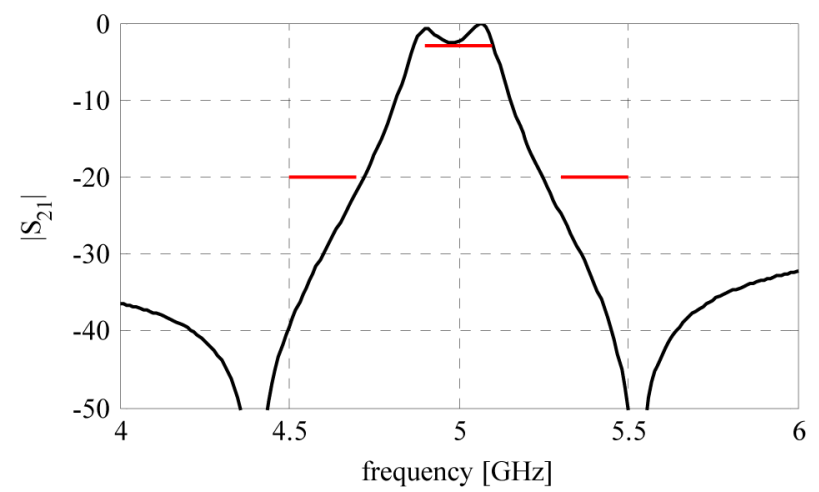

Fig. 11. Microstrip bandpass filter: fine model response $\left(\left|S_{21}\right|\right.$ obtained with Sonnet $\boldsymbol{e m}$ ) at the final design (tuning space mapping with alternative tuning model).

TABLE II

SONNET EM Design PARAMETER VALUeS OF THE Microstrip BandPasS Filter (Tuning Space Mapping With Alternative Tuning Model)

\begin{tabular}{cccc}
\hline $\begin{array}{c}\text { Design } \\
\text { Parameters }\end{array}$ & Initial Solution & $\begin{array}{c}\text { Solution after } \\
\text { the First Iteration }\end{array}$ & $\begin{array}{c}\text { Solution after } \\
\text { the Second Iteration }\end{array}$ \\
\hline$L_{1}$ & $6.00 \mathrm{~mm}$ & $5.333 \mathrm{~mm}$ & $5.362 \mathrm{~mm}$ \\
$L_{2}$ & $6.00 \mathrm{~mm}$ & $6.429 \mathrm{~mm}$ & $6.462 \mathrm{~mm}$ \\
$L_{3}$ & $6.00 \mathrm{~mm}$ & $5.118 \mathrm{~mm}$ & $5.210 \mathrm{~mm}$ \\
$L_{4}$ & $6.00 \mathrm{~mm}$ & $6.971 \mathrm{~mm}$ & $6.785 \mathrm{~mm}$ \\
$g$ & $0.08 \mathrm{~mm}$ & $0.041 \mathrm{~mm}$ & $0.120 \mathrm{~mm}$ \\
\hline \hline
\end{tabular}

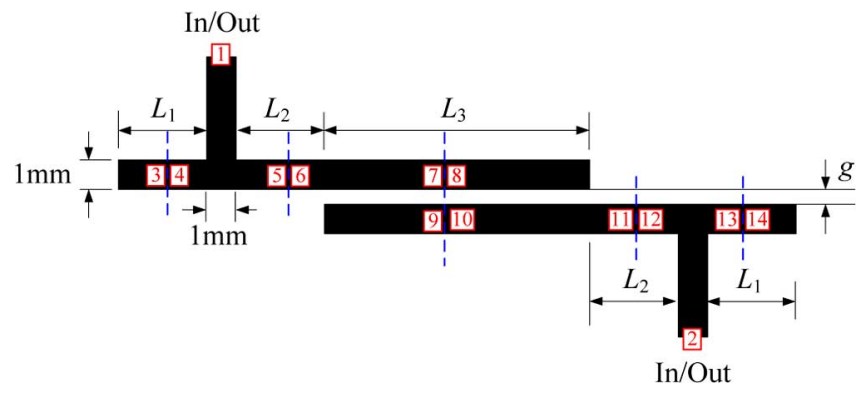

Fig. 12. Second-order tapped-line microstrip filter: physical structure [37].

\section{B. Second-Order Tapped-Line Microstrip Filter [37]}

Our second example is the second-order tapped-line microstrip filter [37] shown in Fig. 12. The design parameters are $x=\left[\begin{array}{llll}L_{1} & L_{2} & L_{3} & g\end{array}\right]^{T}$. Other parameters are fixed at $L_{0}=3.0 \mathrm{~mm}, W=1.0 \mathrm{~mm}, H=0.254 \mathrm{~mm}, \varepsilon_{r}=9.9$, and loss tangent $=0$. The design specifications are

$$
\begin{aligned}
& \left|S_{21}\right| \leq-20 \mathrm{~dB}, \quad \text { for } 3.0 \mathrm{GHz} \leq \omega \leq 4.0 \mathrm{GHz} \\
& \left|S_{21}\right| \geq-3 \mathrm{~dB}, \quad \text { for } 4.75 \mathrm{GHz} \leq \omega \leq 5.25 \mathrm{GHz} \\
& \left|S_{21}\right| \leq-20 \mathrm{~dB}, \quad \text { for } 6.0 \mathrm{GHz} \leq \omega \leq 7.0 \mathrm{GHz} .
\end{aligned}
$$

In this example, the fine model is simulated in Sonnet $\boldsymbol{e m}$, the tuning model is constructed and optimized in Agilent Technologies' ADS. Both the direct and analytical calibration methods are used to implement the calibration process. To construct the tuning model, in Sonnet $\boldsymbol{e m}$, we firstly divide the microstrip sections and the central coupled-line section in the middle and insert co-calibrated ports on the cut-edges. The EM structure with ports is then simulated and the resulting S14P data file is imported into the 14-port $S$-parameter file component in ADS. Ap- 


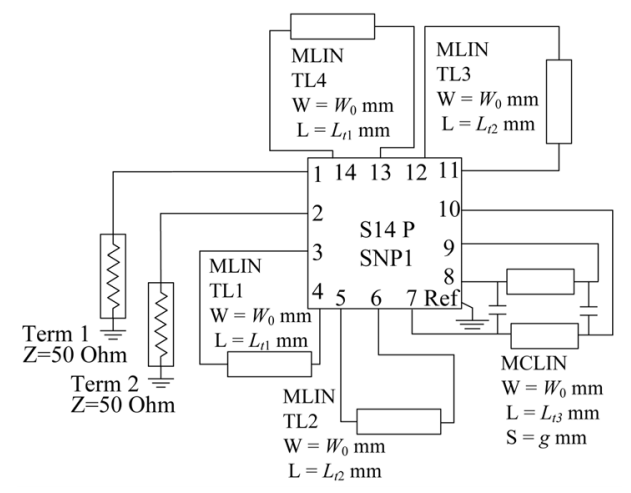

Fig. 13. Second-order tapped-line microstrip filter: tuning model in ADS.

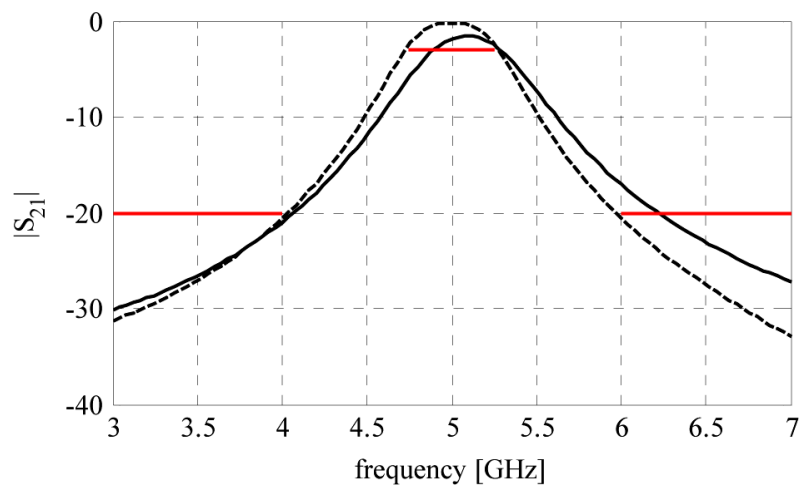

Fig. 14. Second-order tapped-line microstrip filter: fine model response at the initial design (solid line) and the response of the optimized tuning model (dashed line).

propriate circuit components are chosen and attached onto the $S$-parameter file component.

In the ADS circuit simulator, small microstrip components are chosen as the tuning elements to tune the microstrip sections in the EM model, a coupled-line component is used to optimize the central coupled-line polygon in Sonnet. In order to tune the spacing between the coupled lines, we choose two small capacitor components and attach them onto the two sides of the circuit-theory-based coupled-line component. The tuning parameters are thus $\boldsymbol{x}_{t}=\left[\begin{array}{llll}L_{t 1} & L_{t 2} & L_{t 3} & C_{t}\end{array}\right]^{T}$ (lengths in millimeters, capacitance in picofarads) and the tuning model constructed in ADS is shown in Fig. 13.

The initial guess is $\boldsymbol{x}^{(0)}=\left[\begin{array}{llll}3.00 & 4.00 & 2.00 & 0.03\end{array}\right]^{T} \mathrm{~mm}$. In this example, when the tuning parameters are zero, the response of the tuning model is almost the same as the response of the fine model, thus we can get $\boldsymbol{x}_{t .0}^{(i)}=\left[\begin{array}{lllll}0 & 0 & 0 & 0 & 0\end{array}\right]^{T}$.

The tuning model is optimized in ADS with respect to the tuning parameters. The optimal values obtained with (3) are $\boldsymbol{x}_{t .1}^{(0)}=[2.694-2.268-1.497-0.151]^{T}$ mm (lengths in millimeters, capacitance in picofarads). The response of the fine model at the initial guess and the optimized tuning model response are shown in Fig. 14.

In the calibration process, the optimized lengths of the small microstrip components and the small coupled-line component are directly converted to adjustments in the lengths of the microstrip sections and coupled-line section in the EM structure (assuming identity mappings). The spacing between the fine

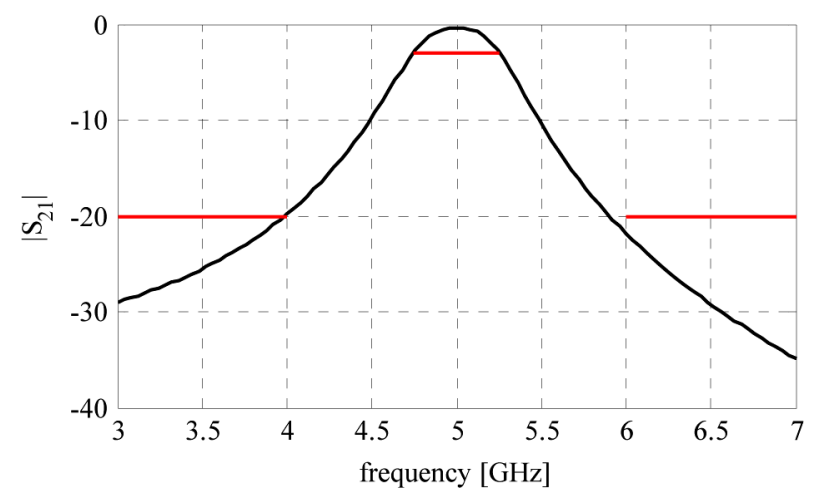

Fig. 15. Second-order tapped-line microstrip filter: fine model response $\left(\left|S_{21}\right|\right.$ obtained with Sonnet $\boldsymbol{e m}$ ) at the final design.

TABLE III

SONNET EM DESIGN PARAMETER VALUES OF THE SECOND-ORdER TAPPED-LINE MICROSTRIP FILTER

\begin{tabular}{ccc}
\hline $\begin{array}{c}\text { Design } \\
\text { Parameters }\end{array}$ & Initial Solution & $\begin{array}{c}\text { Solution after } \\
\text { the First Iteration }\end{array}$ \\
\hline$L_{1}$ & $3.00 \mathrm{~mm}$ & $5.422 \mathrm{~mm}$ \\
$L_{2}$ & $4.00 \mathrm{~mm}$ & $2.012 \mathrm{~mm}$ \\
$L_{3}$ & $2.00 \mathrm{~mm}$ & $4.729 \mathrm{~mm}$ \\
$g$ & $0.03 \mathrm{~mm}$ & $0.138 \mathrm{~mm}$ \\
\hline \hline
\end{tabular}

model's coupled-line polygons is adjusted according to the optimal value of the capacitor using the analytical calibration method. To implement this analytical calibration, the initial design $\boldsymbol{x}^{(0)}$ is perturbed with respect to $g$ by $\Delta g=0.01 \mathrm{~mm}$, and the corresponding change $\Delta C_{t}$ of $C_{t}$ is found for which the fine model response at the above perturbed design is matched by the tuning model response at $\boldsymbol{x}_{t .0}^{(0)}$ perturbed by $\Delta C_{t}$, which is $\Delta C_{t}=-0.0091 \mathrm{pF}$. Since the perturbation is very small, we can assume that there is a linear mapping between the change of the capacitance and the change of the spacing. The coefficient is calculated as $s=\Delta g / \Delta C_{t}=-1.099 \mathrm{~mm} / \mathrm{pF}$.

The new design obtained after calibration is $\boldsymbol{x}^{(1)}=$ $\left[\begin{array}{lllll}5.333 & 6.429 & 5.118 & 6.791 & 0.406\end{array}\right]^{T} \mathrm{~mm}$ and this solution satisfies the design specifications. Fig. 15 shows the fine model response at $\boldsymbol{x}^{(1)}$. The values of the design variables are summarized in Table III.

\section{High-Temperature Superconducting (HTS) Filter}

Fig. 16 illustrates the structure of an HTS filter. The design parameters are the lengths of the coupled-line sections and the spacing between them, which are shown as $L_{1}, L_{2}, L_{3}, S_{1}, S_{2}, S_{3}$, respectively. The width of all the sections is $W=0.178 \mathrm{~mm}$ and the length of the input and output microstrip-line sections is $L_{0}=1.27 \mathrm{~mm}$. A substrate of lanthanum aluminate is used with $\varepsilon_{r}=23.425$, height $H=0.508 \mathrm{~mm}$, and loss tangent $=0.00003$. The metallization is considered lossless. The design specifications are

$$
\begin{array}{ll}
\left|S_{21}\right| \leq 0.05, & \text { for } \omega \leq 3.967 \mathrm{GHz} \\
\left|S_{21}\right| \geq 0.95, & \text { for } 4.008 \mathrm{GHz} \leq \omega \leq 4.058 \mathrm{GHz} \\
\left|S_{21}\right| \leq 0.05, & \text { for } \omega \geq 4.099 \mathrm{GHz} .
\end{array}
$$

The tuning model is constructed by dividing the five coupled-line polygons in the middle and inserting the tuning ports at the new cut edges. Its S22P data file (22 being the number 


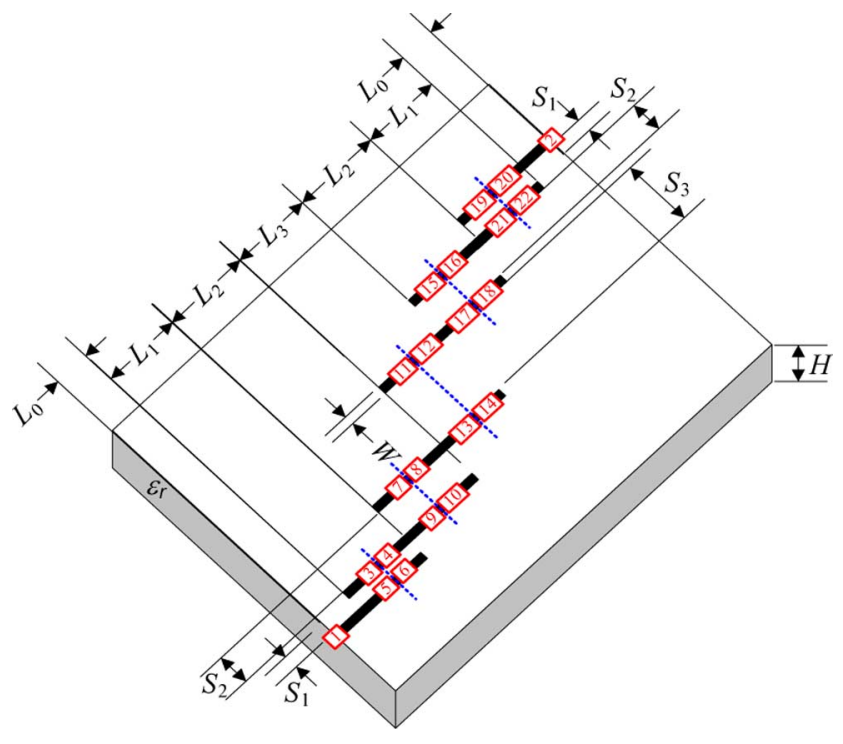

Fig. 16. HTS filter: physical structure [38].

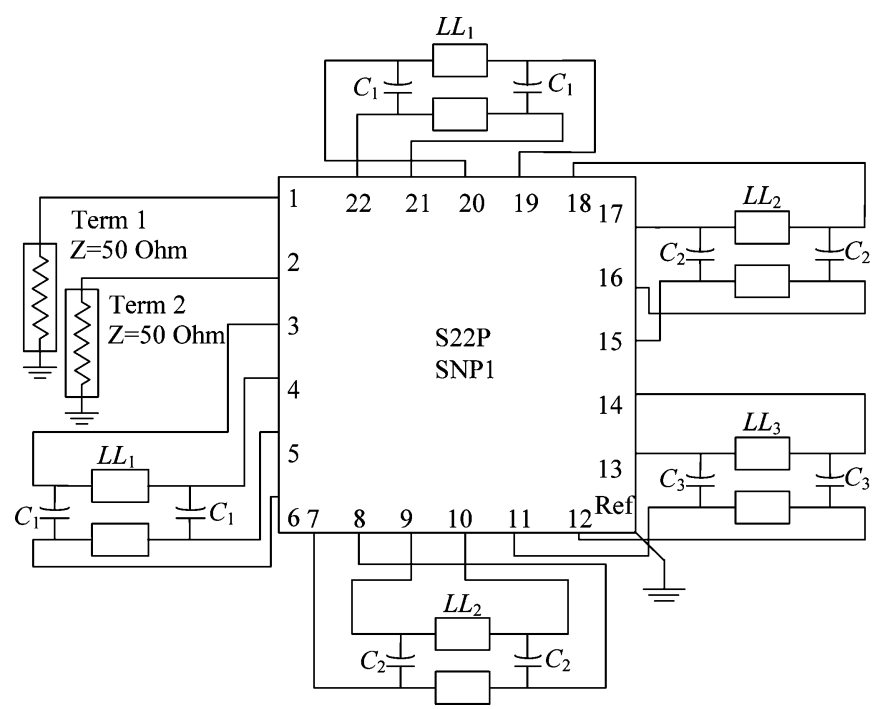

Fig. 17. HTS filter: tuning model (Agilent Technologies' ADS).

of ports) is then loaded into the $S$-parameter component in $\mathrm{Ag}$ ilent Technologies' ADS. The circuit-theory coupled-line components and capacitor components are chosen to be the tuning elements and are inserted into each pair of tuning ports (Fig. 17). The lengths of the imposed coupled lines and the capacitances of the capacitors are assigned to be the tuning parameters so that we have $x_{t}=\left[\begin{array}{llllll}L L_{1} & L L_{2} & L L_{3} & C_{1} & C_{2} & C_{3}\end{array}\right]^{T}\left(L L_{k}\right.$ in millimeters, $C_{k}$ in picofarads).

The calibration model is implemented in ADS and shown in Fig. 18. It contains the same tuning elements as the tuning model. It basically mimics the division of the coupled lines performed while preparing $\boldsymbol{R}_{t}$. The calibration model also contains six (implicit) space-mapping parameters that will be used as parameters $\boldsymbol{p}$ in the calibration process (6), (7). These parameters are $\boldsymbol{p}=\left[\begin{array}{llllll}H_{1} & H_{2} & H_{3} & \varepsilon_{r 1} & \varepsilon_{r 2} & \varepsilon_{r 3}\end{array}\right]^{T}$, where $H_{k}$ and $\varepsilon_{r k}$ are substrate height (in millimeters) and dielectric constant of the coupled-line segment of length $L_{k}$

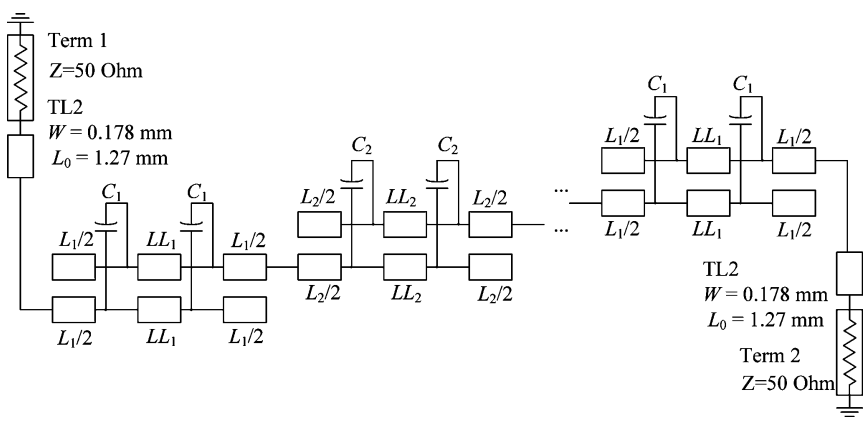

Fig. 18. HTS filter: calibration model (Agilent Technologies' ADS).

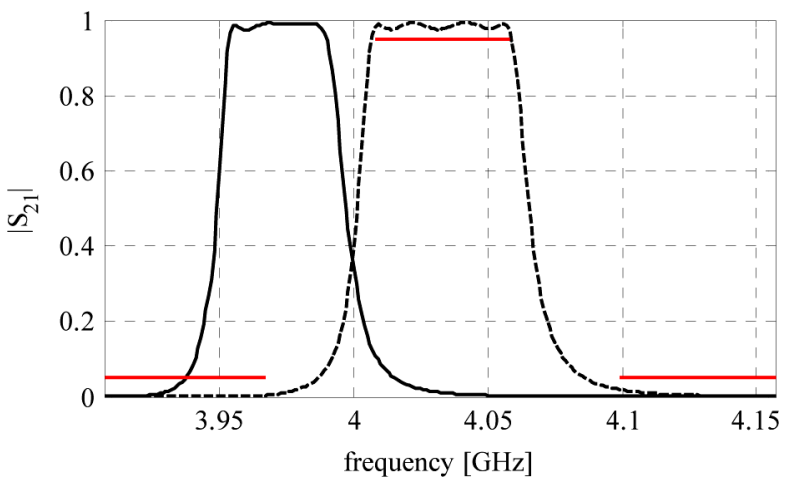

Fig. 19. HTS filter: fine model response at the initial design (solid line) and the response of the optimized tuning model (dashed line).

according to Fig. 16. Initial values of these parameters are $\left[\begin{array}{llllll}0.508 & 0.508 & 0.508 & 9.8 & 9.8 & 9.8\end{array}\right]^{T}$.

The initial design, $\boldsymbol{x}^{(0)}=\left[\begin{array}{llll}4.806 & 4.984 & 4.803 & 0.561\end{array}\right.$ $2.3932 .698]^{T} \mathrm{~mm}$, is the optimal solution of the coarse model, i.e., the calibration model with zero values of the tuning parameters.

In this example, there is a small misalignment between the fine model response and the tuning model response with the tuning elements set to zero, even though the tuning elements have been inserted using co-calibrated ports. Therefore, the alignment process (2) gives nontrivial values of

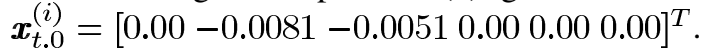

Fig. 19 shows the fine model response at the initial solution, and the response of the optimized tuning model. The tuning parameters obtained with (3) are

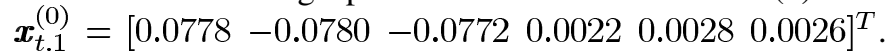
Note that some of the parameters take negative values, which is permitted in ADS.

Now, the calibration process must be performed in order to find the updated values of the design parameters. First, the space-mapping parameters are adjusted using (6) to align the calibration model with the optimized tuning model for the values of tuning parameters equal $\boldsymbol{x}_{t .0}^{(0)}$. We get $\boldsymbol{p}^{(0)}=\left[\begin{array}{llllll}0.488 & 0.409 & 0.371 & 25.1 & 23.2 & 24.9\end{array}\right]^{T}$. The new design $x^{(1)}=\left[\begin{array}{llllll}4.653 & 4.971 & 4.638 & 0.544 & 2.055 & 2.174\end{array}\right]^{T} \mathrm{~mm}$ is then found using (7). This solution already satisfies the design specifications; however, we perform a second tuning space-mapping iteration to improve it further. The final design

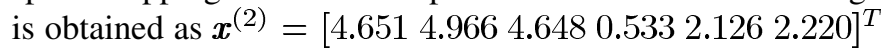
mm. Fig. 20 shows the fine model response at $\boldsymbol{x}^{(2)}$. The values 


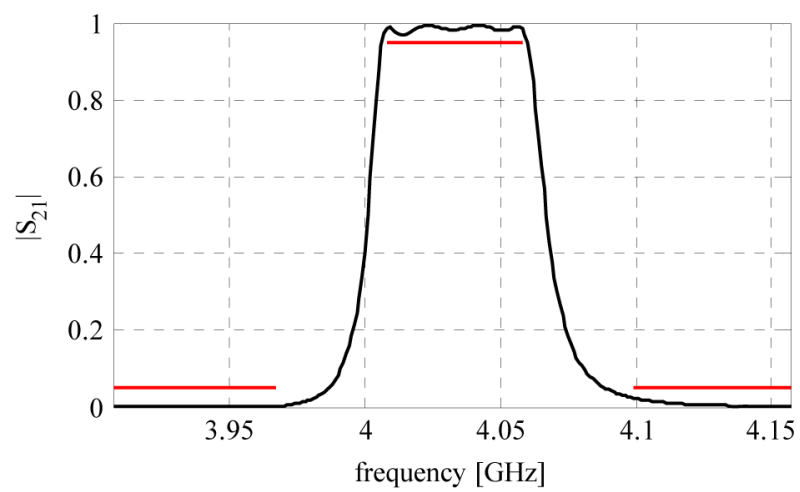

Fig. 20. HTS filter: fine model response $\left(\left|S_{21}\right|\right.$ obtained with Sonnet $\left.\boldsymbol{e m}\right)$ at the final design.

TABLE IV

SONNET EM DESIGN PARAMETER VALUES OF TUNING SPACE-MAPPING METHOD FOR THE HTS FILTER DESIGN

\begin{tabular}{cccc}
\hline \hline $\begin{array}{c}\text { Design } \\
\text { Parameters }\end{array}$ & Initial Solution & $\begin{array}{c}\text { Solution after } \\
\text { the First Iteration }\end{array}$ & $\begin{array}{c}\text { Solution after } \\
\text { the Second Iteration }\end{array}$ \\
\hline$L_{1}$ & $4.806 \mathrm{~mm}$ & $4.653 \mathrm{~mm}$ & $4.651 \mathrm{~mm}$ \\
$L_{2}$ & $4.984 \mathrm{~mm}$ & $4.971 \mathrm{~mm}$ & $4.966 \mathrm{~mm}$ \\
$L_{3}$ & $4.803 \mathrm{~mm}$ & $4.638 \mathrm{~mm}$ & $4.648 \mathrm{~mm}$ \\
$S_{1}$ & $0.561 \mathrm{~mm}$ & $0.544 \mathrm{~mm}$ & $0.533 \mathrm{~mm}$ \\
$S_{2}$ & $2.393 \mathrm{~mm}$ & $2.055 \mathrm{~mm}$ & $2.126 \mathrm{~mm}$ \\
$S_{3}$ & $2.698 \mathrm{~mm}$ & $2.174 \mathrm{~mm}$ & $2.220 \mathrm{~mm}$ \\
\hline \hline
\end{tabular}

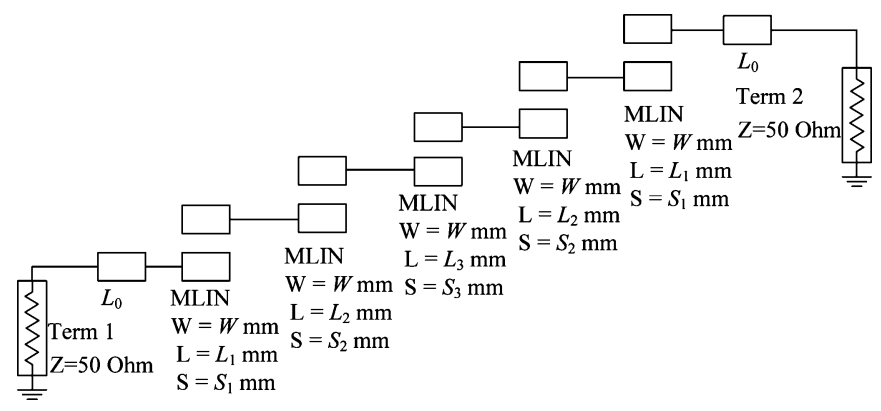

Fig. 21. HTS filter: implicit space-mapping coarse model (Agilent Technologies' ADS).

of the design variables are summarized in Table IV. Note that the tuning space-mapping algorithm requires only one iteration to satisfy the design specifications, and only one additional iteration to obtain an almost equal-ripple fine model response.

To have a comparison with the novel tuning space-mapping algorithm, we implement this HTS filter again using implicit space-mapping method. The fine model is exactly the same as the one used in the implicit space mapping [2]. Agilent Technologies' ADS is selected to be the circuit simulator to construct the coarse model. As is shown in Fig. 21, the ADS coarse model consists of empirical models for single and coupled microstrip transmission lines with ideal open stubs.

The preassigned parameters are heights and dielectric constants of the coupled-line sections in the coarse model. Thus, the preassigned parameter vector is $\boldsymbol{p}=$ $\left[\begin{array}{llllll}H_{1} & H_{2} & H_{3} & \varepsilon_{r 1} & \varepsilon_{r 2} & \varepsilon_{r 3}\end{array}\right]^{T}$. The implicit space-mapping algorithm requires two iterations to satisfy the design specification $\left(\boldsymbol{x}^{(1)}=\left[\begin{array}{llllll}4.788 & 4.857 & 4.778 & 0.594 & 2.106 & 2.250\end{array}\right]^{T}\right.$ $\left.\mathrm{mm} ; \boldsymbol{x}^{(2)}=\left[\begin{array}{llllll}4.785 & 4.846 & 4.780 & 0.589 & 2.111 & 2.266\end{array}\right]^{T} \mathrm{~mm}\right)$, while tuning space mapping requires only one iteration. The major reason for the effectiveness of the tuning space-mapping method is that the tuning model comprises information from the fine model simulation result, while this data is not contained in the implicit space-mapping coarse model.

\section{CONCLUSION}

A novel and efficient technique for microwave design is presented, which brings together the engineering concept of tuning with the efficiency of space mapping. We present the formal description of the tuning space-mapping algorithm and discuss the calibration procedure, as well as the practical aspects of our methodology. A simple example illustrating the concept of the tuning space mapping is provided that explains the construction of the tuning model and both the analytical and surrogate-based calibrations. Several microwave design optimization problems are considered that verify the robustness of the proposed approach.

\section{ACKNOWLEDGMENT}

The authors thank Sonnet Software Inc., Syracuse, NY, for making $\boldsymbol{e m}$ available, and Agilent Technologies, Santa Rosa, $\mathrm{CA}$, for making ADS available.

\section{REFERENCES}

[1] J. W. Bandler, R. M. Biernacki, S. H. Chen, P. A. Grobelny, and R. H. Hemmers, "Space mapping technique for electromagnetic optimization," IEEE Trans. Microw. Theory Tech., vol. 42, no. 12, pp. 536-544, Dec. 1994.

[2] J. W. Bandler, Q. S. Cheng, N. K. Nikolova, and M. A. Ismail, "Implicit space mapping optimization exploiting preassigned parameters," IEEE Trans. Microw. Theory Tech., vol. 52, no. 1, pp. 378-385, Jan. 2004.

[3] J. W. Bandler, Q. S. Cheng, S. A. Dakroury, A. S. Mohamed, M. H. Bakr, K. Madsen, and J. Sondergaard, "Space mapping: The state of the art," IEEE Trans. Microw. Theory Tech., vol. 52, no. 1, pp. 337-361, Jan. 2004.

[4] S. Koziel, J. W. Bandler, and K. Madsen, "A space mapping framework for engineering optimization: Theory and implementation," IEEE Trans. Microw. Theory Tech., vol. 54, no. 10, pp. 3721-3730, Oct. 2006.

[5] S. Koziel and J. W. Bandler, "Space-mapping optimization with adaptive surrogate model," IEEE Trans. Microw. Theory Tech., vol. 55, no. 3, pp. 541-547, Mar. 2007.

[6] D. Echeverria and P. W. Hemker, "Space mapping and defect correction," Int. Math. J. Comput. Methods App. Math., vol. 5, no. 2, pp. 107-136, 2005.

[7] M. A. Ismail, D. Smith, A. Panariello, Y. Wang, and M. Yu, "EMbased design of large-scale dielectric-resonator filters and multiplexers by space mapping," IEEE Trans. Microw. Theory Tech., vol. 52, no. 1, pp. 386-392, Jan. 2004.

[8] K.-L. Wu, Y.-J. Zhao, J. Wang, and M. K. K. Cheng, "An effective dynamic coarse model for optimization design of LTCC RF circuits with aggressive space mapping," IEEE Trans. Microw. Theory Tech., vol. 52, no. 1, pp. 393-402, Jan. 2004

[9] S. Amari, C. LeDrew, and W. Menzel, "Space-mapping optimization of planar coupled-resonator microwave filters," IEEE Trans. Microw. Theory Tech., vol. 54, no. 5, pp. 2153-2159, May 2006.

[10] M. Dorica and D. D. Giannacopoulos, "Response surface space mapping for electromagnetic optimization," IEEE Trans. Magn., vol. 42, no. 4, pp. 1123-1126, Apr. 2006.

[11] J. E. Rayas-Śnchez and V. Gutiérrez-Ayala, "EM-based Monte Carlo analysis and yield prediction of microwave circuits using linear-input neural-output space mapping," IEEE Trans. Microw. Theory Tech., vol. 54, no. 12, pp. 4528-4537, Dec. 2006.

[12] S. J. Leary, A. Bhaskar, and A. J. Keane, "A constraint mapping approach to the structural optimization of an expensive model using surrogates," Optim. Eng., vol. 2, no. 4, pp. 385-398, Dec. 2001.

[13] M. Redhe and L. Nilsson, "Using space mapping and surrogate models to optimize vehicle crashworthiness design," in 9th AIAA/ISSMO Multidisciplinary Anal. Optim. Symp., Atlanta, GA, Sep. 2002, Paper AIAA-2002-5536. 
[14] H.-S. Choi, D. H. Kim, I. H. Park, and S. Y. Hahn, "A new design technique of magnetic systems using space mapping algorithm," IEEE Trans. Magn., vol. 37, no. 5, pp. 3627-3630, Sep. 2001.

[15] L. Encica, J. Makarovic, E. A. Lomonova, and A. J. A. Vandenput, "Space mapping optimization of a cylindrical voice coil actuator," IEEE Trans. Ind. Appl., vol. 42, no. 6, pp. 1437-1444, Nov.-Dec. 2006.

[16] N. M. Alexandrov and R. M. Lewis, "An overview of first-order model management for engineering optimization," Optim. Eng., vol. 2, no. 4, pp. 413-430, Dec. 2001.

[17] A. J. Booker, J. E. Dennis, Jr., P. D. Frank, D. B. Serafini, V. Torczon, and M. W. Trosset, "A rigorous framework for optimization of expensive functions by surrogates," Struct. Optim., vol. 17, no. 1, pp. 1-13, Feb. 1999

[18] S. J. Leary, A. Bhaskar, and A. J. Keane, "A knowledge-based approach to response surface modeling in multifidelity optimization," Global Optim., vol. 26, no. 3, pp. 297-319, Jul. 2003.

[19] S. E. Gano, J. E. Renaud, and B. Sanders, "Variable fidelity optimization using a kriging based scaling function," in Proc. 10th AIAA/ISSMO Multidisciplinary Anal. Optim. Conf., Albany, NY, 2004, pp. 1-19, AIAA-2004-4460.

[20] T. W. Simpson, J. Peplinski, P. N. Koch, and J. K. Allen, "Metamodels for computer-based engineering design: Survey and recommendations," Eng. Comput., vol. 17, no. 2, pp. 129-150, Jul. 2001.

[21] N. V. Queipo, R. T. Haftka, W. Shyy, T. Goel, R. Vaidynathan, and P. K. Tucker, "Surrogate-based analysis and optimization," Progr. Aerosp. Sci., vol. 41, no. 1, pp. 1-28, Jan. 2005.

[22] S. Koziel, J. W. Bandler, A. S. Mohamed, and K. Madsen, "Enhanced surrogate models for statistical design exploiting space mapping technology," in IEEE MTT-S Int. Microw. Symp. Dig., Long Beach, CA, Jun. 2005, pp. 1609-1612.

[23] S. Koziel, J. W. Bandler, and K. Madsen, "Theoretical justification of space-mapping-based modeling utilizing a data base and on-demand parameter extraction," IEEE Trans. Microw. Theory Tech., vol. 54, no. 12, pp. 4316-4322, Dec. 2006.

[24] S. Koziel and J. W. Bandler, "Microwave device modeling using space-mapping and radial basis functions," in IEEE MTT-S Int. Microw. Symp. Dig., Honolulu, HI, 2007, pp. 799-802.

[25] V. K. Devabhaktuni, B. Chattaraj, M. C. E. Yagoub, and Q.-J. Zhang, "Advanced microwave modeling framework exploiting automatic model generation, knowledge neural networks, and space mapping," IEEE Trans. Microw. Theory Tech., vol. 51, no. 7, pp. 1822-1833, Jul. 2003.

[26] J. E. Rayas-Sánchez, "EM-based optimization of microwave circuits using artificial neural networks: The state-of-the-art," IEEE Trans. Microw. Theory Tech., vol. 52, no. 1, pp. 420-435, Jan. 2004.

[27] J. E. Rayas-Sánchez, F. Lara-Rojo, and E. Martinez-Guerrero, "A linear inverse space-mapping (LISM) algorithm to design linear and nonlinear RF and microwave circuits," IEEE Trans. Microw. Theory Tech., vol. 53, no. 3, pp. 960-968, Mar. 2005.

[28] L. Zhang, J. Xu, M. C. E. Yagoub, R. Ding, and Q.-J. Zhang, "Efficient analytical formulation and sensitivity analysis of neuro-space mapping for nonlinear microwave device modeling," IEEE Trans. Microw. Theory Tech., vol. 53, no. 9, pp. 2752-2767, Sep. 2005.

[29] S. Koziel, J. W. Bandler, and K. Madsen, "Towards a rigorous formulation of the space mapping technique for engineering design," in Proc. Int. Circuits Syst. Symp., Kobe, Japan, May 2005, pp. 5605-5608.

[30] K. Madsen and J. Søndergaard, "Convergence of hybrid space mapping algorithms," Optim. Eng., vol. 5, no. 2, pp. 145-156, Jun. 2004.

[31] J. C. Rautio, "RF design closure-Companion modeling and tuning methods," presented at the IEEE MTT-S Int. Microw. Symp. Workshop, San Francisco, CA, 2006.

[32] D. G. Swanson and R. J. Wenzel, "Fast analysis and optimization of combline filters using FEM," in IEEE MTT-S Int. Microw. Symp. Dig., Boston, MA, Jul. 2001, pp. 1159-1162.

[33] J. Meng, S. Koziel, J. W. Bandler, M. Bakr, and Q. S. Cheng, "Tuning space mapping: A novel technique for engineering design optimization," in IEEE MTT-S Int. Microw. Symp. Dig., Atlanta, GA, 2008, pp. 991-994.

[34] $\boldsymbol{e m}$. ver. 11.52, Sonnet Softw. Inc., North Syracuse, NY, 2007.

[35] Advanced Design System (ADS). ver. 2003C, Agilent Technol., Santa Rosa, CA, 2003.

[36] A. Hennings, E. Semouchkina, A. Baker, and G. Semouchkin, "Design optimization and implementation of bandpass filters with normally fed microstrip resonators loaded by high-permittivity dielectric," IEEE Trans. Microw. Theory Tech., vol. 54, no. 3, pp. 1253-1261, Mar. 2006.

[37] A. Manchec, C. Quendo, J.-F. Favennec, E. Rius, and C. Person, "Synthesis of capacitive-coupled dual-behavior resonator (CCDBR) filters," IEEE Trans. Microw. Theory Tech., vol. 54, no. 6, pp. 2346-2355, Jun. 2006.
[38] J. W. Bandler, R. M. Biernacki, S. H. Chen, R. H. Hemmers, and K. Madsen, "Electromagnetic optimization exploiting aggressive space mapping," IEEE Trans. Microw. Theory Tech., vol. 43, no. 12, pp. 2874-2882, Dec. 1995.

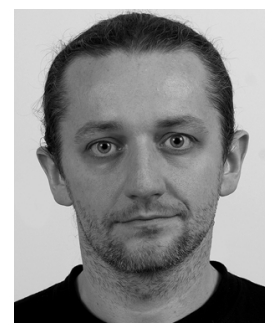

Slawomir Koziel (M'03-SM'07) received the M.Sc. and $\mathrm{Ph} . \mathrm{D}$. degrees in electronic engineering from Gdansk University of Technology, Gdansk, Poland, in 1995 and 2000, respectively, and the M.Sc. degrees in theoretical physics and mathematics and Ph.D. degree in mathematics from the University of Gdansk, Gdansk, Poland, in 2000, 2002, and 2003, respectively.

He is currently with the School of Science and Engineering, Reykjavík University, Reykjavík, Iceland. He has authored or coauthored over 120 papers. His research interests include surrogate-based modeling and optimization, space mapping, circuit theory, analog signal processing, evolutionary computation, and numerical analysis.

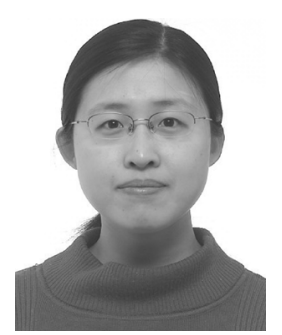

Jie Meng received the B.Eng. degree in electrical engineering from the University of Science and Technology of China, Hefei, China, in 2004, and the M.A.Sc. degree in electrical engineering from McMaster University, Hamilton, ON, Canada, in 2008.

From 2004 to 2006, she was with the Semiconductor Manufacturing International Corporation, Beijing, China. In September 2006, she joined the Simulation Optimization Systems Research Laboratory, McMaster University, as a Research Assistant. Her research interests include CAD, RF and microwave circuit design and modeling, optimization theory and algorithms, space-mapping and surrogate model optimization, and EM field simulation and optimization.

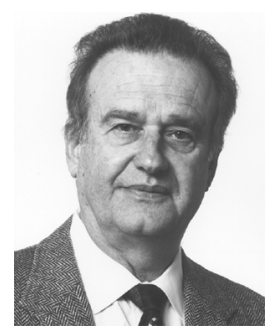

John W. Bandler (S'66-M'66-SM'74-F'78LF'06) studied at Imperial College. He received the B.Sc. (Eng.), Ph.D., and D.Sc. (Eng.) degrees from the University of London, London, U.K., in 1963, 1967, and 1976, respectively

In 1969, he joined McMaster University, Hamilton, ON, Canada. He is currently a Professor Emeritus. He was President of Optimization Systems Associates Inc., which he founded in 1983, until November 20, 1997, the date of acquisition by the Hewlett-Packard Company. He is currently President of Bandler Corporation, Dundas, ON, Canada, which he founded in 1997.

Dr. Bandler is a Fellow of several societies including the Royal Society of Canada. He was the recipient of the 2004 IEEE Microwave Theory and Techniques Society (IEEE MTT-S) Microwave Application Award.

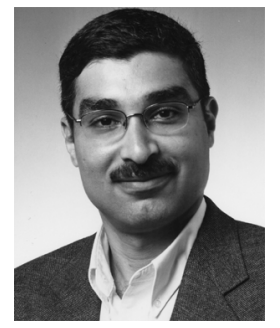

Mohamed H. Bakr (S'98-M'00) received the B.Sc. degree in electronics and communications engineering (with distinction) and M.Sc. degree in engineering mathematics from Cairo University, Cairo, Egypt, in 1992 and 1996, respectively, and the $\mathrm{Ph} . \mathrm{D}$. degree from McMaster University, Hamilton, ON, Canada, in 2000.

In November 2000, he joined the Computational Electromagnetics Research Laboratory (CERL), University of Victoria, Victoria, BC, Canada, as a Natural Sciences and Engineering Research Council of Canada (NSERC) Post-Doctoral Fellow. He is currently an Associate Professor with the Department of Electrical and Computer Engineering, McMaster University. His research areas include CAD and modeling of microwave circuits and photonic devices, neural-network applications, and biolelectromagnetism. 


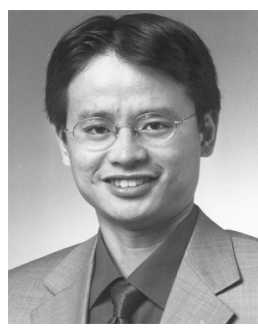

Qingsha S. Cheng (S'00-M'05) was born in Chongqing, China. He received the B.Eng. and M.Eng. from Chongqing University, Chongqing, China, in 1995 and 1998, respectively, and the Ph.D. degree from McMaster University, Hamilton, ON, Canada, in 2004.

In 1998, he joined the Department of Computer Science and Technology, Peking University, Beijing, China. In 1999, he joined the Department of Electrical and Computer Engineering, McMaster University, where he is currently a Research Associate with the Department of Electrical and Computer Engineering and a Lecturer with the Faculty of Engineering. His research interests are surrogate modeling, CAD, modeling of microwave circuits, software design technology, and methodologies for microwave $\mathrm{CAD}$. 